\title{
CRECIMIENTO INDUSTRIAL Y RIESGO URBANO: EL CASO DE MEXICALI, BAJA CALIFORNIA*
}

\author{
Por \\ Pablo Jesús González Reyes**
}

\begin{abstract}
RESUMEN
El presente articulo consiste en una valoración de situaciones de riesgo ambiental relacionadas con incendios, explosiones y emanaciones tóxicas, debidas al creciente manejo de substancias químicas en la industria manufacturera del área urbana de Mexicali, Baja California. La metodología incluye la utilización de sistemas de información geográfica. Con el uso de esta herramienta se identifican con claridad diversas áreas de riesgo en la ciudad y se proponen, además, algunas alternativas para la toma de decisiones.
\end{abstract}

\begin{abstract}
This article consists on an assessment of environmental risk situations related to fires, explotions, and toxic releases, due to the increacing handling of chemical substances in the manufacturing industry located in the urban area of Mexicali, Baja California. The methodology includes the use of geographic information systems. Several high-risk areas were identified in the city, and altenatives are proposed, as an aid in decision-making.
\end{abstract}

\section{INTRODUCCIÓN ${ }^{1}$}

En las últimas tres décadas la frontera norte del país, en la cual se sitúa la ciudad de Mexicali, Baja California, ha sido escenario de un complejo proceso demográfico y económico. Con el desarrollo de la actividad industrial apuntalado por el programa de maquiladoras, la zona fronteriza ha logrado una progresiva integración a la economía de los Estados Unidos, y en años recientes, la atracción de inversiones asiáticas como parte de la dinámica de internacionalización del capital que hoy se vive en el mundo entero.

* Este artículo fue recibido en junio de 1996 para su publicación en la revista Estudios Fronterizos.

** Investigador del Instituto de Investigaciones Sociales de la Universidad Autónoma de Baja California.

Este trabajo es la versión modificada de una ponencia presentada en la reunión anual de la Association of Bordeland Scholars en Reno, Nevada, en abril de 1996. 
Pero uno de los aspectos más cuestionados de este desarrollo industrial es el impacto ecológico negativo que produce al incidir de manera perjudicial en el medio ambiente y la salud de la población fronteriza. Este planteamiento fue el argumento principal esgrimido por los grupos ambientalistas del sur de los Estados Unidos para exigir que se incluyera dentro del Tratado de Libre Comercio un acuerdo adicional de cooperación ambiental entre México y ese país vecino.

La utilización de crecientes volúmenes de substancias químicas peligrosas en la industria fronteriza, además de los daños generales que provocan a los ecosistemas (aire, agua, etcétera), convierten a este sector económico en un elemento crítico en cuanto a las probabilidades de ocurrencia de siniestros ocasionados por incendios, explosiones y emanaciones tóxicas, con la consiguiente vulnerabilidad de la población trabajadora de las industrias de alto riesgo, al igual que de la población que habita en las áreas aledañas a esas empresas, y en las zonas cercanas a las vialidades por donde se transportan las substancias tóxicas.

Asimismo, el crecimiento desordenado de las ciudades fronterizas contribuye de manera fundamental en la agudización del riesgo y la vulnerabilidad de la población en casos de accidentes en la industria relacionados con substancias tóxicas peligrosas, debido a los conflictos derivados de las incompatibilidades en el uso del suelo urbano, ante la ausencia de un eficaz ordenamiento territorial en la ciudad.

Sin duda que el establecimiento ascendente de maquiladoras es motivo de preocupación ante el incremento de las probabilidades de riesgo químico. Los cambios estructurales ocurridos en los últimos años en la industria manufacturera ${ }^{2}$ fronteriza en relación con la incorporación cada vez mayor de empleos en la industria electrónica, eléctrica, y metalmecánica, las cuales predominan en cuanto al uso de substancias químicas peligrosas en el proceso productivo y en sus fases auxiliares, hacen de esta actividad un factor de alto riesgo poblacional, razón por la cual se requiere

2 El INEG define como manufacturas a :

los procesos de trabajo mediante los cuales se transforman materias primas en productos nuevos, ya sea con actividades manuales, procesos químicos o con ayuda de maquinaria, para obtener bienes que satisfagan las necesidades de la sociedad... Quedan comprendidas dentro de estas actividades las maquiladoras industriales, que son aquellos establecimientos que realizan, de manera exclusiva, la fabricación, ensamble, y actividades de transformación en general, cuya producción final no es de su propiedad, sino que pertenecen y se destinan en su totalidad a otros establecimientos que le aportan las materias primas y auxiliares, así como el diseño y requerimiento de dicha producción. También se incluyen las actividades referentes a la refinación del petróleo y a la petroquímica básica. (INEGI, 1984). 
de mecanismos más eficientes de seguridad industrial y control que permitan evitar desastres de lamentables consecuencias.

\section{ORÍGENES DE LA ACTIVIDAD INDUSTRIAL EN MEXICALI}

La ciudad de Mexicali, Baja California, surgió prácticamente al inicio del presente siglo. Sus primeros signos de vida industrial se dejaron ver hasta la década de 1920, mediante actividades relacionadas con la producción de alimentos y cerveza. Para entonces, el valle de Mexicali comenzaba a perfilarse internacionalmente como un área fértil para la agricultura, dadas las bondades geográficas que ofrece a la región las aguas del Río Colorado. En la década de 1930, la actividad industrial empezó a vincularse al sector agrícola y agropecuario de la región, además de impulsar la producción de vinos y licores, aguas gaseosas, entre otros. (Sández, 1988:187-188).

El desarrollo agrícola del valle de Mexicali en las décadas de 1940 y 1950, aunado al inicio del Programa de Braceros en ese periodo, fueron factores decisivos para el crecimiento urbano de Mexicali, al convertirse la región en un polo de atracción poblacional proveniente del interior de la República Mexicana. En el lapso de 1940 a 1960, la población de Mexicali se incremento casi diez veces al pasar de 18,775 a 179,539 habitantes. (VI y VIII Censos generales de población).

Al igual que Mexicali, el resto de las principales ciudades de la frontera norte del país manifestaron una dinámica de población creciente. Con la caída del precio internacional del algodón y la terminación del Programa de Braceros, se empezaron a presentar agudos problemas de desempleo al rebasar la demanda de mano de obra la oferta del mercado laboral. En esas circunstancias surge en la década de 1960 el Programa de Industrialización Fronteriza (PIF), el cual dio cabida a un importante flujo de inversion extranjera a través del establecimiento de las maquiladoras.

Así, a partir de los años sesenta se inicia en la franja fronteriza norte del país una etapa decisiva de impulso al crecimiento industrial, que se fue reflejando en una presencia mayor del sector secundario en la economía como fuente importante de generación de empleos para la región.

\section{ESTRUCTURA INDUSTRIAL DE MEXICALI}

En el caso particular de Mexicali, la industria manufacturera ocupaba en 1950 sólo el 8\% de la población económicamente activa (PEA), pero en 1990 el porcentaje había ascendido a $18.5 \%$ (VII y XI Censos generales de población y vivienda). En este último año la ciudad contaba con poco más de medio millon de habitantes. 
CUADRO 1. Personal ocupado promedio en la industria manufacturera de Mexicali, Baja California.

\begin{tabular}{lccccc}
\hline Subsector & 1970 & 1975 & 1985 & 1988 & 1993 \\
\hline $\begin{array}{l}\text { Productos alimenticios, } \\
\text { bebidas y tabaco }\end{array}$ & 22.5 & 21.9 & 21.2 & 10.6 & 18.5 \\
$\begin{array}{l}\text { Textiles, prendas de vestir } \\
\text { e industria del cuero }\end{array}$ & 23.5 & 19.4 & 14.5 & 14.2 & 6.0 \\
$\begin{array}{l}\text { Productos metálicos, } \\
\text { maquinaria y equipo* }\end{array}$ & 23.6 & 44.9 & 47.0 & 55.2 & 55.7 \\
$\begin{array}{l}\text { Otros } \\
\text { Total }\end{array}$ & 30.4 & 13.8 & 17.3 & 20.0 & 19.8 \\
\hline
\end{tabular}

FUENTE: 1970-1975 Sández, Avilés y Álvarez (1993:24); 1985-1993 INEGI.

* Incluye rama eléctrica, electrónica y transporte.

Cabe resaltar que en el periodo de las décadas de 1970 hasta principios de 1990 sucedieron importantes cambios estructurales en el sector de la industria manufacturera de Mexicali. En el cuadro 1 se aprecia que en 1970, la distribución del personal ocupado promedio no era significativamente diferente en los subsectores ahí especificados. Sin embargo, en lo que respecta a la producción de alimentos, bebidas y tabaco se observa una constante disminución en la participación de empleos hasta 1988, lográndose una recuperación para 1993 al llegar a Mexicali inversiones destinadas a ese giro industrial.

En el caso de la industria textil, resulta interesante notar la importancia cada vez menor que tiene este subsector en cuanto a su contribución en la generación de empleos dentro de la industria manufacturera. Para 1993, el subsector representó únicamente el $6 \%$ de los empleos manufactureros. En cambio el subsector productos metálicos, maquinaria y equipo ha sido el más activo desde la década de los setenta, además de concentrar desde fines de la década pasada más de la mitad de los empleos generados en la industria de la manufactura.

Sin duda que el dinamismo observado en la industria manufacturera y las transformaciones estructurales del sector, responden en gran parte a la presencia de la industria maquiladora en la frontera norte del país. En el caso de Mexicali, durante los años de 1985, 1989 y 1993, la maquiladora 
absorbi6 el $53 \%, 87 \%$ y $61 \%$ de los empleos generados en el sector manufacturero, respectivamente. ${ }^{3}$

\section{INDUSTRIALIZACIÓN Y MEDIO AMBIENTE}

La industria maquiladora se instaló en México a mediados de los años sesenta, dando con ello inicio a grandes flujos de inversion extranjera en las principales ciudades fronterizas del norte del país. En un periodo de sólo diez años - de 1965 a 1975 - las maquiladoras ya ocupaban a sesenta mil obreros, con lo cual prácticamente se convirtieron en el símbolo de la industria fronteriza, pues pese a que únicamente representaban la décima parte del total de plantas manufactureras, absorbían casi las tres cuartas partes de la planta laboral incorporada a la industria de la manufactura (Tamayo, 1992:10-11).

$\mathrm{Si}$ bien en los inicios del programa maquilador predominaba la rama textil, posteriormente se desarrollaron los subsectores eléctrico, electrónico y el metal-mecánico. Estos cambios estructurales de la industria maquiladora adquirieron suma importancia para la ecología regional, en virtud de que los nuevos subsectores utilizan en sus procesos productivos grandes volúmenes de substancias químicas peligrosas. A partir de los años ochenta se incrementaron las importaciones de estas substancias químicas, elevando con ello los niveles de riesgo ambiental y de salud en la población (Tamayo,1992:16).

En la actualidad, Mexicali ocupa a nivel nacional el tercer lugar en cuanto al número de maquiladoras instaladas, encabezadas por Tijuana y Ciudad Juárez. En el cuadro 2 se observa el comportamiento de las maquiladoras establecidas en Mexicali, notándose un incremento considerable a partir de 1986, con algunos altibajos en los años posteriores, debido principalmente a la recesión de la economía estadunidense.

Con la entrada en vigor del Tratado de Libre Comercio (TLC), se tiene prácticamente asegurada la continuidad en el dinamismo de la actividad industrial en la frontera norte y en otras regiones del país con ventajas locacionales. Empero, esta nueva etapa coyuntural significa enormes presiones al medio ambiente y mayores riesgos químicos asociados con la actividad industrial en esas regiones económicas.

Con base en informacion reciente, se obtuvo que del total de nuevas maquiladoras establecidas en México en 1995 (334 plantas), el mayor

3 Estos porcentajes se calcularon a partir del promedio anual de empleos que registra INEGI en los Censos Económicos, y en los reportes periódicos que publica sobre el comportamiento de la industria maquiladora de exportación. 
porcentaje correspondió a Baja California (23\%), seguida por Chihuahua y Durango con $10 \%$ y $8 \%$ respectivamente. Cabe resaltar que Mexicali ocupo el tercer lugar en la lista de ciudades favorecidas con nuevas maquiladoras, correspondiendo los primeros dos lugares a Tijuana y Gómez Palacio (El Economista,14 de diciembre de 1995:3,6 y 7). En otras palabras, las ventajas locacionales que otorga la ubicación geográfica de Baja California comienzan a reflejar un mayor potencial en la generación de empleos manufactureros como resultado de la aplicación del TLC.

La inminente apertura de otra aduana fronteriza Mexicali-Caléxico es un indicador de la dinámica económica y poblacional que se vive en esta region, en donde se requiere el fomento de una infraestructura que permita afrontar los retos de una mayor integración a la economía de los Estados Unidos. Si bien el nuevo puerto fronterizo abre la posibilidad de incrementar la capacidad de movilización de mercancías requeridas en los procesos industriales tanto de Mexicali como de otras localidades del estado (Tijuana, Tecate, Ensenada), también plantea el riesgo que implica el transporte de mayores volúmenes de substancias tóxicas peligrosas en la ciudad.

CUADRO 2. Características de la industria maquiladora de exportación en Mexicali, Baja California.

\begin{tabular}{cccccc}
\hline Año & $\begin{array}{c}\text { Núm. de } \\
\text { establec. }\end{array}$ & $\begin{array}{c}\text { Personal } \\
\text { ocup. prom. }\end{array}$ & Año & $\begin{array}{r}\text { Núm. de } \\
\text { establec. }\end{array}$ & $\begin{array}{c}\text { Personal } \\
\text { ocup. prom. }\end{array}$ \\
\hline 1975 & 67 & 6324 & 1986 & 86 & 12727 \\
1976 & 69 & 6604 & 1987 & 109 & 16312 \\
1977 & 70 & 6351 & 1988 & 135 & 19558 \\
1978 & 65 & 6543 & 1989 & 147 & 21374 \\
1979 & 77 & 7965 & 1990 & 139 & 21724 \\
1980 & 79 & 7146 & 1991 & 131 & 20846 \\
1981 & 64 & 7628 & 1992 & 139 & 19905 \\
1982 & 54 & 6268 & 1993 & 140 & 20528 \\
1983 & 55 & 7392 & 1994 & 124 & 21452 \\
1984 & 67 & 10264 & $1995 *$ & 119 & 24188 \\
1985 & 75 & 10876 & & & \\
\hline
\end{tabular}

FUENTE: INEGI.

* Hasta julio. 


\section{RIESGO QUÍMICO URBANO}

El Plan Estatal de Desarrollo Urbano de Baja California menciona tres clases de riesgos que ponen en condiciones de vulnerabilidad los asentamientos humanos de la region: los fenómenos geológicos (sismos), los hidrometeorologicos (inundaciones, trombas, etcétera), y los químicos. Entre estos últimos se destacan "...los incendios de todo tipo, las intoxicaciones provocadas por industrias con riesgo químico y la incidencia de contingencias causadas por la industria petroquímica." (Periódico oficial del Estado de Baja California, 24 de junio de 1994:44).

El crecimiento industrial de Mexicali ha significado a partir de los años sesenta, y especialmente desde la década de 1980, mayores niveles de riesgo y vulnerabilidad para la población urbana en cuanto a siniestros causados por incendios, explosiones, o emanaciones de gases tóxicos asociados con la actividad productiva de la industria de transformación.

$\mathrm{Al}$ incremento de industrias manufactureras que desarrollan actividades de alto riesgo ${ }^{4}$ se suman también las empresas de servicios que manejan substancias químicas, pues por su nivel de toxicidad o el volumen almacenado en plena área urbana de la ciudad, representan un alto riesgo para la población. Entre ellas se destacan la planta de PEMEX, la planta central de distribución y venta de gas butano-propano, al igual que múltiples empresas dedicadas a la venta y comercialización de insecticidas y pesticidas. Consecuentemente, la frecuencia de los accidentes químicos se ha empezado a manifestar en Mexicali. A continuación se mencionan algunos ejemplos:

En 1988 una fuga del gasoducto que pasa por la periferia sur de la ciudad provocó dos muertes al estallar una concentración de gasolina gasificada. En la misma zona, casi ocho años después, se presentó otra fuga de gasolina de mayores proporciones, pero afortunadamente la oportuna evacuación de la población evitó que se presentara una severa catástrofe. (La Crónica de Baja California, 21 de enero de1996).

En 1990 una fuga de ácido sulfúrico en la planta Química Orgánica obligó a la evacuación de varios miles de habitantes de las áreas aledañas

\footnotetext{
4 En este trabajo se consideran actividades de alto riesgo aquellas que se ciñen a los criterios establecidos por el gobierno federal, ya que su regulación es asunto de alcance general en la nación y de interés para la federación (Art. 5 de la Ley general de equilibrio ecológico y de protección del ambiente). Estos criterios se especifican, por un lado, en el listado de actividades de alto riesgo que las instituciones de gobierno publican en el Diario oficial de la federación; por el otro, en la normatividad técnica que utiliza la Secretaría de Salud para definir el grado de riesgo sanitario en los establecimientos, cuya observancia es indispensable para el otorgamiento de autorizaciones sanitarias.
} 
a la empresa, reportándose múltiples casos de personas intoxicadas por el nocivo agente tóxico. (Sánchez, 1991:565).

También en 1990 se presentó una fuga de amoniaco en los ductos de gas del sistema de refrigeración de una empresa pasteurizadora localizada en un área residencial céntrica de la ciudad, provocando intoxicaciones y la necesidad de evacuación de los residentes cercanos al lugar del siniestro. (La Voz de la Frontera, 6 de junio de 1990).

En 1992 se suscitó un incendio en una fábrica productora de papel, que por el manejo de volúmenes de substancias tóxicas está calificada por las autoridades de salud y el departamento de bomberos como empresa que desarrolla actividades de alto riesgo. (La Voz de la Frontera, 21 de junio de 1992).

Al inicio del presente año se produjo una fuga de amoniaco en una empresa que comercializa fertilizantes, al reventarse una manguera cuando se trasegaba el producto químico. Esto ocasionó la evacuación del personal y el cierre temporal de la transitada carretera Mexicali-San Luis Río Colorado, al cubrirse el área de una extensa nube de gas. (La Crónica de Baja California, 10 de enero de 1996).

Unos meses después se suscita una explosión en el almacén de una planta maquiladora al estallar diversos tibores que contenían substancias químicas como thinner, flux, acetona, alcohol, isopropil, entre otras, las cuales son altamente flamables. De nuevo la evacuación del personal y de vecinos residentes aledaños a la fábrica evitaron pérdidas humanas. ( $\mathrm{La}$ Voz de la Frontera, 28 de mayo de 1996).

Por fortuna, hasta el momento no han ocurrido accidentes de mayores proporciones a los ya relatados; sin embargo, perdura en la mente de los mexicanos los trágicos sucesos ocurridos en 1984 en San Juanico y 1992 en Guadalajara, los cuales son pruebas contundentes de los riesgos y la vulnerabilidad a que está expuesta la población urbana ante la utilización creciente de substancias químicas peligrosas en un ambiente de laxitud en la aplicación de la normatividad jurídica, la corrupción y las deficiencias en la planeación urbana.

La transportación intraurbana de substancias químicas peligrosas representa un riesgo potencial para algunas áreas geográficas de la ciudad de Mexicali. Así lo indican los casos de accidentes ocurridos en diversos puntos del área urbana, que ponen en peligro la salud e incluso la vida de la población. Sin duda que a mayor dinamismo en la actividad industrial de alto riesgo habrá también mayor vulnerabilidad en lugares críticos para el tránsito vehicular y en las zonas de densidad poblacional cercanas a las principales vialidades de la ciudad. 
Los crecientes volúmenes de substancias químicas peligrosas utilizadas en la actividad económica industrial de Mexicali han generado la preocupación e interés de diversos sectores de la población, que de diferentes formas manifiestan su temor y repudio ante las autoridades de gobierno para exigir la reubicación de empresas que representan un peligro para la seguridad física de los habitantes de la ciudad ( $\mathrm{La} \mathrm{Voz} \mathrm{de} \mathrm{la}$ Frontera, 10 de junio de 1990).

En años recientes se integró el Grupo de Respuesta a Emergencias Químicas, dependiente del Departamento de Bomberos y Protección Civil de la ciudad de Mexicali, cuyo equipo de trabajo se adquirió con base en donativos de la Cámara Nacional de la Industria de Transformación local. Asimismo, se creó un convenio internacional de colaboración para casos de emergencias químicas. (La Crónica de Baja California, 18 de febrero de 1996).

Esas acciones si bien se inscriben dentro de los lineamientos generales estratégicos planteados en el Programa Frontera XxI, ${ }^{5}$ instrumento de la política binacional ambiental para la frontera México-Estados Unidos, no dejan de ser en el contexto de las ciudades fronterizas una parte mínima e insuficiente ante el enorme reto que implica la necesidad de garantizar la seguridad de su población.

\section{CRECIMIENTO URBANO Y LOCALIZACIÓN INDUSTRIAL}

$\mathrm{Al}$ igual que sucede en el resto de las ciudades del país, Mexicali se ha desarrollado históricamente bajo la ausencia de una adecuada planeación que regule su crecimiento. La expansión un tanto anárquica de la ciudad ocasiona múltiples problemas de funcionalidad urbana.

Ante estas circunstancias surge de manera inevitable una mezcla indiscriminada en el uso del suelo urbano que revela incompatibilidades en el ordenamiento territorial de la ciudad. Un ejemplo típico es la localización de industrias en espacios que corresponden predominantemente a áreas de uso residencial, de servicios, recreativos, o de otro uso específico, creando con ello situaciones diversas de riesgo ambiental y de conflicto con la población aledaña.

Con la creación de parques industriales desde fines de los sesenta, y mas aún en los ochenta, con orientación hacia la periferia de las ciudades en virtud del atractivo precio del suelo, comenzaron a manifestarse notables

5 Entre los antecedentes de cooperación ambiental efectuados por México y Estados Unidos en torno a la frontera norte, destacan el Convenio de la Paz de 1983, el Plan Integral Ambiental Fronterizo (PIAP), y el Programa Frontera XXI de 1995, el cual retoma la experiencia adquirida con el PIAF. 
cambios en la localización de la actividad industrial urbana a lo largo de la frontera norte. Esta novedad en la distribución espacial de la industria fronteriza surgí a raíz de una serie de programas promovidos por el gobierno federal para ofrecer áreas de arrendamiento a las plantas maquiladoras. (Álvarez y Mungaray, 1986:180).

Si bien la relocalización de plantas maquiladoras representó un importante avance en la sectorización espacial de la industria, el crecimiento sostenido de la población fronteriza y su distribución en la mancha urbana, ha conducido a que algunos parques industriales compartan estrecha vecindad con áreas residenciales de reciente creación; quedando sujetas éstas a una situación de mayor vulnerabilidad ante el alto riesgo químico que implica habitar en áreas cercanas a estas concentraciones industriales.

Por otra parte, la creación de parques industriales ha resultado atractivo para muchas empresas que se instalan en ellos; otras, sin embargo, prefieren continuar operando fuera de aquellos porque así se lo indica su lógica de acumulación de capital, de manera que el conflicto de riesgo químico que generan con la población aledaña se mantiene latente.

Como señalan Álvarez y Mungaray,

[1]a actual localización de la industria maquiladora dentro de los espacios urbanos de las ciudades fronterizas, indican en cierta forma la subordinación de la calidad de vida de la población a los objetivos del crecimiento económico logrado a través de la maquiladora. (Álvarez y Mungaray, 1986:182).

En la actualidad existen en Mexicali un total de once parques industriales (figura 1), de los cuales la mayoría se crearon en la década de 1980 en las áreas periféricas de la ciudad. Esta expansión del espacio industrial en la ciudad revela la confianza depositada en el futuro desarrollo industrial de la región.

\section{RIESGO QUÍMICO, VULNERABILIDAD POBLACIONAL Y SISTEMAS DE INFORMACIÓN GEOGRÁFICA}

Los avances científicos y tecnológicos logrados en las últimas décadas en áreas como la computación, cartografía y teledetección han sido claves para el desarrollo de sistemas de información geográfica. ${ }^{6}$ La creciente

6 Existen diversas definiciones sobre los sistemas de información geográfica. En este trabajo los entendemos como aquellos sistemas computarizados para la captura, almacenamiento, revisión, manipulación, análisis y representación visual de información espacial georeferenciada. 


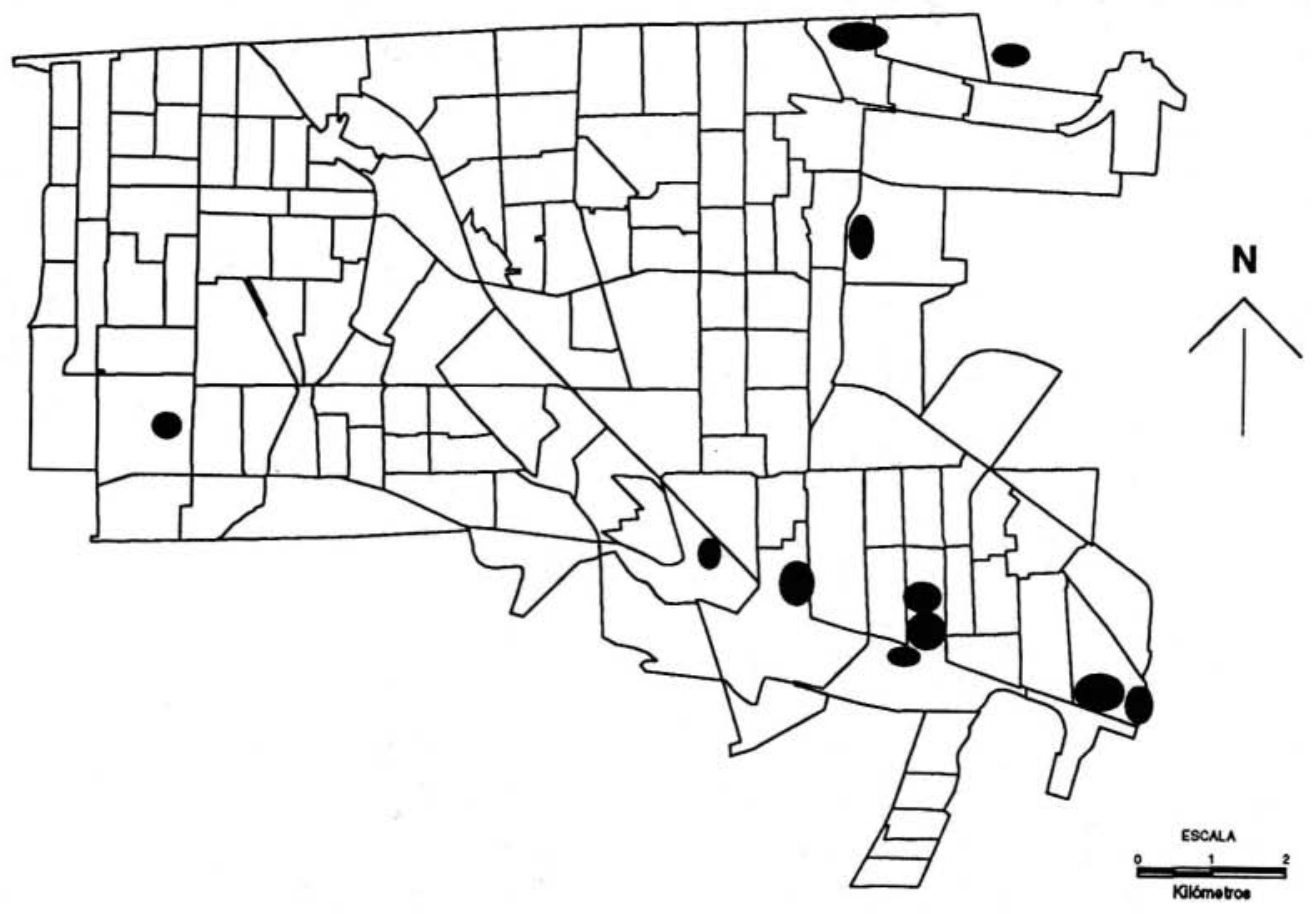

Figura 1. Localización geográfica de los parques industriales de Mexicali, B.C. FUENTE: Instituto de investigaciones Sociales, UABC. 
incorporación de estos sistemas en el sector privado, de gobierno y académico se ha extendido a nivel internacional a través de una amplia gama de aplicaciones. (Star,b1990; Huxhold, 1991; Elliot, 1995). Una de ellas es el uso de sistemas de información geográfica para el análisis del manejo de substancias químicas peligrosas utilizadas en los procesos productivos de la industria de transformación. (Estes, 1987; McMaster, 1990; Fedra, 1989).

La preocupación por los problemas ambientales derivados del aumento poblacional y la industrialización de la frontera norte del país ha motivado en años recientes la necesidad de generar un sistema binacional de información geográfica ambiental para la frontera México-Estados Unidos. (Wright, Hepner y Metzner, 1993). Bajo esta misma perspectiva se procedio a utilizar las herramientas técnico-metodologicas que ofrecen los sistemas de información geográfica, para hacer una valoración exploratoria de los niveles de riesgo y vulnerabilidad poblacional en la ciudad de Mexicali, asociados con la utilización de substancias químicas peligrosas en los procesos productivos - y sus fases auxiliares - de la industria manufacturera que opera en la ciudad.

Partiendo de información obtenida en la Secretaría de Salud y el Departamento de Bomberos y Protección Civil en Mexicali, se identificó la localización geográfica de los establecimientos manufactureros de la ciudad calificados como de alto riesgo con base en su actividad y volumen de substancias tóxicas utilizadas. Estas planitas manufactureras incluyeron a 32 maquiladoras y 21 industrias no maquiladoras. Como puede apreciarse en la figura 2, la ubicación urbana de esas empresas se distribuye tanto en áreas periféricas de la ciudad como en lugares cercanos al centro tradicional de la ciudad. Si bien la industria maquiladora tiende a concentrarse en los parques industriales, en la industria no maquiladora se observa una concentración en el centro tradicional y en la parte sureste de la ciudad.

Cabe destacar que el $85 \%$ de las maquiladoras de alto riesgo pertenecen a las ramas eléctrica y electrónica (47\%), así como a la metal-mecánica (38\%). Esto es importante ya que ambas ramas operan con insumos que incluyen una diversidad de substancias tóxicas tales como ácidos, substancias alcalinas, pinturas, solventes, flux, adhesivos, barnices, resinas, entre otras. (Sánchez, 1989:163).

En lo que respecta a la industria no maquiladora, la cual incluye el $40 \%$ del total de empresas de alto riesgo en la ciudad, las substancias químicas

7 Un estudio pionero en la aplicación de esta metodología para el análisis de riesgos químicos industriales en la frontera México-Estados Unidos es el excelente trabajo de Roberto A. Sánchez aplicado a la industria maquiladora de Mexicali. (Sánchez, 1991). 


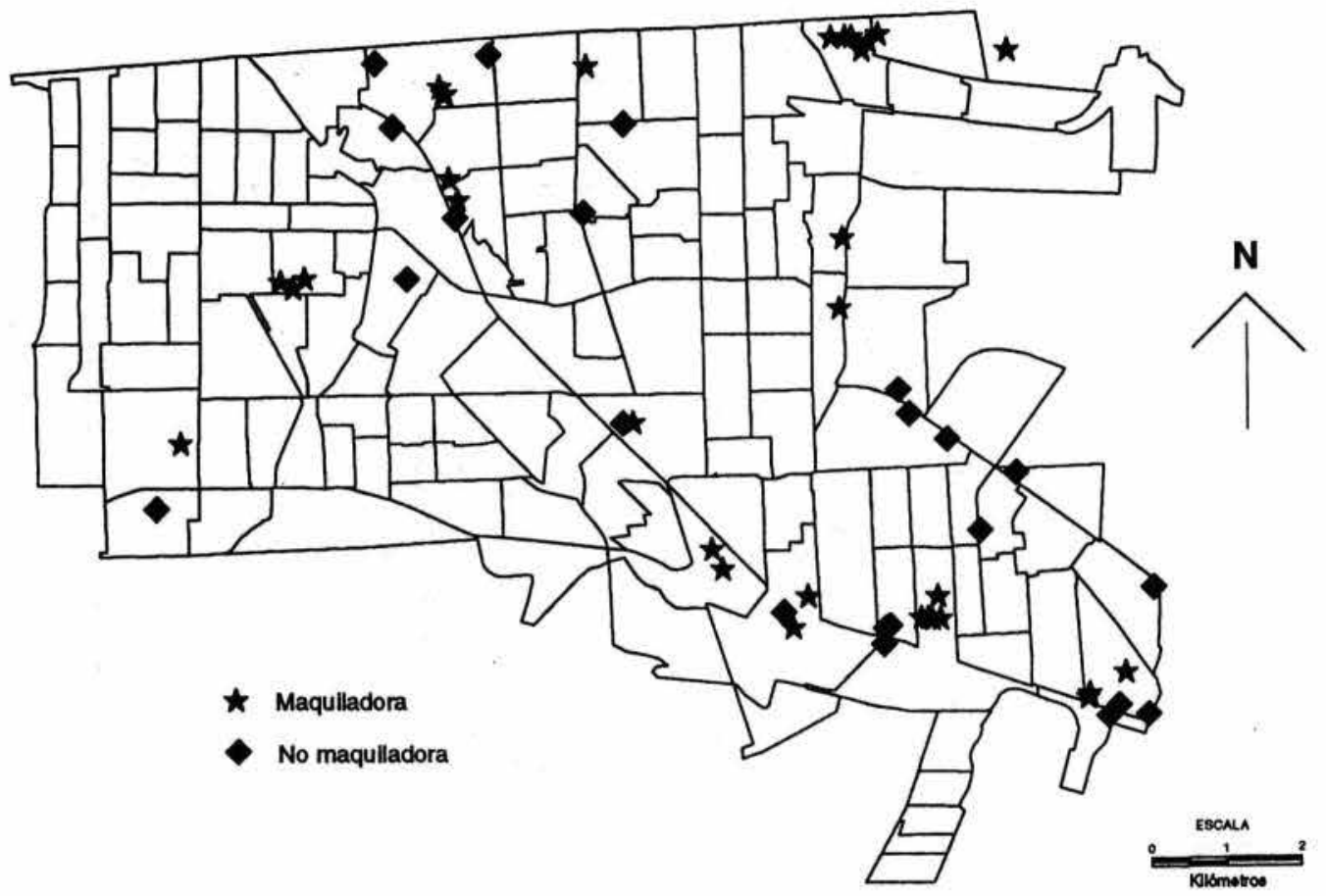

Figura 2. Distribución espacial de industrias de alto riesgo en Mexicali, B.C. FUENTE: Instituto de Investigaciones Sociales, UABC. 
tóxicas predominantes son el amoniaco, gas industrial (metano-propano), y acetileno. En el caso del primero, el riesgo consiste en que es un gas sumamente irritante, y los otros dos en grandes volúmenes representan un enorme peligro en caso de incendio y explosión.

Con base en la información que proporciona la Cámara Nacional de la Industria de Transformación local se obtuvo que poco más de las tres cuartas partes (76\%) de los empleos generados en las empresas de alto riesgo se ubican en industrias maquiladoras. Del total de empleos en este último sector $(15,291)$, el $82 \%$ laboraba en las ramas eléctrica, electrónica y metal-mecánica. (CANACINTRA, 1995).

La figura 3 nos muestra la distribución de la población urbana por áreas geoestadísticas básicas (AGEBS). Pueden apreciarse aglomeraciones de industrias de alto riesgo en zonas de la ciudad de alta y mediana densidad poblacional, lo cual las convierte en áreas vulnerables a siniestros causados por riesgos químicos. Cabe destacar que el área céntrica de la ciudad, así como la parte noreste y sureste de Mexicali concentran industrias de alto riesgo en zonas de alta densidad de población.

En la figura 4 se pueden apreciar las principales vías de flujo vehicular de la ciudad, a partir de corredores con anchura de 250 metros de distancia respecto a la vialidad, notándose que a lo largo de ellas se sitúan la mayoría de las industrias de alto riesgo. Este dato es importante porque permite identificar áreas vulnerables de Mexicali en cuanto al tránsito de substancias tóxicas peligrosas, sobre todo si las relacionamos con las zonas de más alta densidad poblacional de la ciudad. En el sector sureste de la ciudad se observan concentraciones de industrias de alto riesgo en dos importantes vialidades que se ubican hacia la periferia oriente de Mexicali, mismas que se conectan con la carretera federal a San Luis Río Colorado, Sonora; es decir, son vínculos de comunicación terrestre de la ciudad con el resto de la república mexicana.

En la figura 5 se identificaron las escuelas de Mexicali que se encuentran ubicadas a una distancia no mayor de quinientos metros con respecto a alguna empresa de alto riesgo. En total son 54 escuelas cuya proximidad con áreas de actividad industrial de riesgo las convierten en zonas vulnerables a sinietros provocados por agentes químicos industriales. Éste es el caso especialmente de las escuelas localizadas en la parte céntrica y en el sector noreste de la ciudad.

Por último, la figura 6 muestra la distribución de servicios médicos públicos y de bomberos en Mexicali. Puede apreciarse, por un lado, que los servicios médicos públicos se concentran en la zona céntrica de la ciudad, lo cual representa un problema de distancia y tiempo en caso de suscitarse un siniestro ocasionado por agentes químicos industriales, dada 


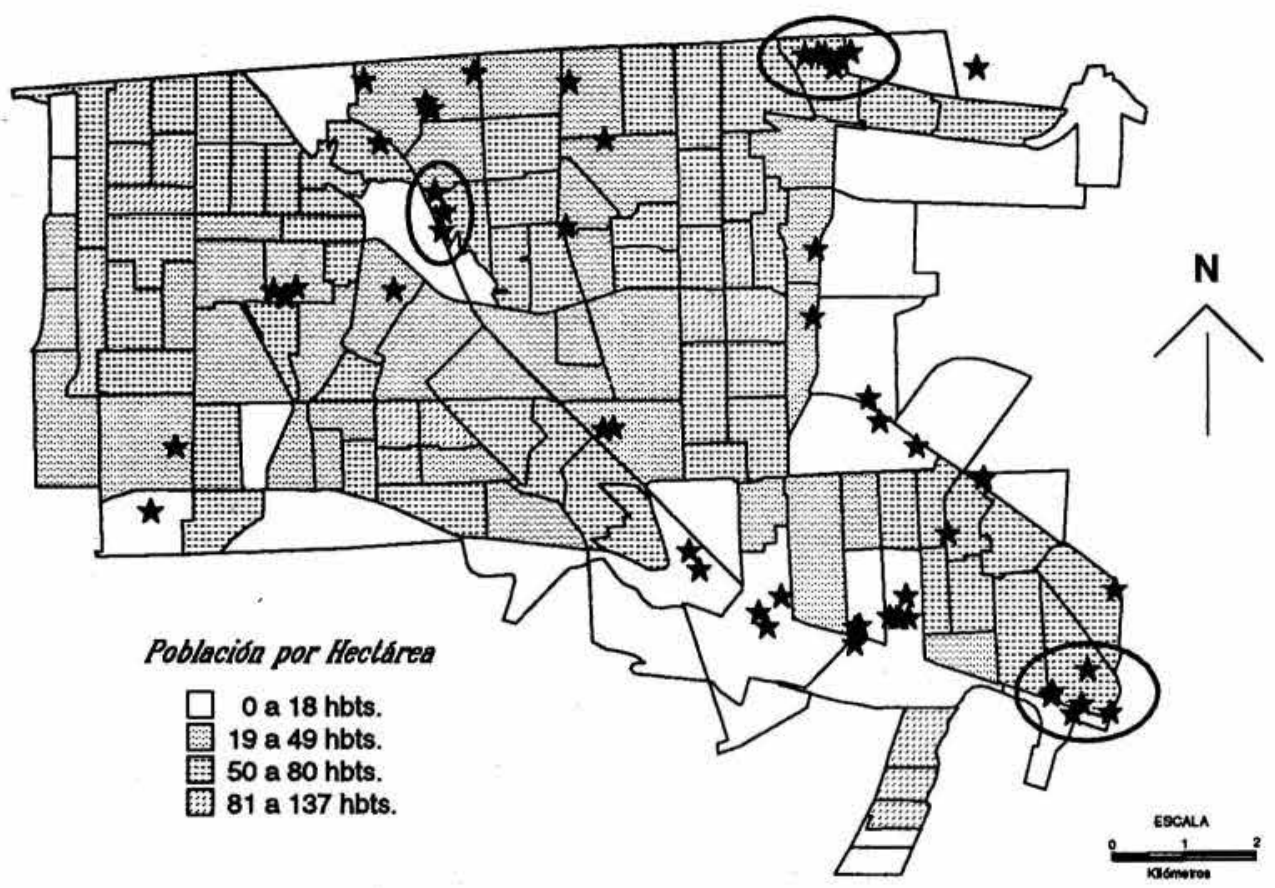

Figura 3. Distribución espacial de industrias de alto riesgo y densidad poblacional, Mexicali, B.C. FUENIE: Instituto de investigaciones Sociales, UABC. 


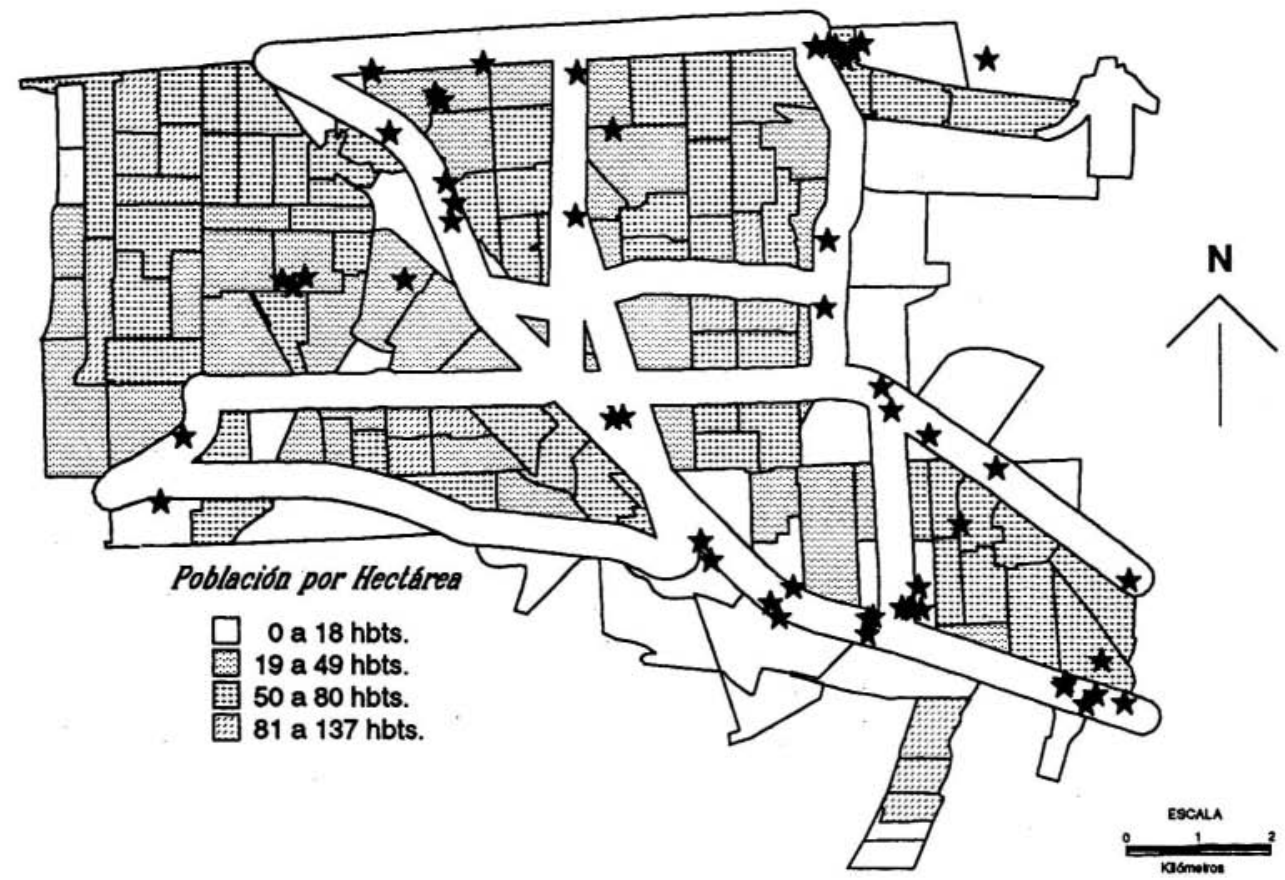

Figura 4. Principales rutas de transporte de substancias tóxicas en Mexicali, B.C. FUENTE: Instituto de investigaciones Sociales, UABC. 
la necesidad de trasladar heridos de emergencia, sobre todo si el accidente ocurre en el sector oriente de la ciudad. Por otro lado, el número de estaciones de bomberos y los recursos técnicos disponibles han sido rebasados por el crecimiento urbano de Mexicali, ya que de las seis estaciones de bomberos, en cuatro hay sólo una bombera.

\section{CONCLUSIONES}

La dinámica de crecimiento industrial y demográfico de Mexicali ha incrementado los niveles de riesgo y vulnerabilidad de la población en casos de siniestros ocasionados por el manejo de substancias tóxicas industriales. Si bien es cierto que la industria manufacturera $-\mathrm{y}$ en especial la maquiladora- cumplen una función de gran relevancia como fuentes generadoras de empleo, y con grandes expectativas de ampliarse aún más en el futuro inmediato, también lo es la necesidad de evitar que ocurran accidentes, e incluso catástrofes, cuyas probabilidades de ocurrir aumentan en la medida que se elevan los volúmenes de substancias químicas peligrosas en el área urbana de la ciudad.

En la actualidad es de carácter urgente que los tres niveles de gobierno, municipal, estatal y federal, en coordinación con los diversos sectores sociales interesados en las actividades industriales, participen de manera decisiva en la construcción de estrategias encaminadas a prevenir accidentes químicos de origen industrial. Al respecto, mucho se puede avanzar a nivel de relocalización de industrias de alto riesgo, en la observancia del ordenamiento territorial y uso de suelo evitando el exceso de proximidad de áreas residenciales en zonas industriales, en la aplicación de medidas más eficientes de seguridad industrial, en la elaboración de programas educativos en el campo de la protección civil y su impartición en escuelas y a la comunidad en general, en la aplicación efectiva de medidas que regulen el transporte de substancias químicas por la ciudad, etcétera.

Finalmente, se considera indispensable disponer de un programa eficiente de contingencias que permita garantizar a la población de la ciudad mayor seguridad en la capacidad de respuesta a accidentes químicos industriales. Estas acciones contribuirían a que Mexicali se coloque en mejores condiciones de afrontar los difíciles desafíos de desarrollo ambiental y de salud poblacional que le exige el crecimiento económico de la región, ya que de no actuar oportuna y atinadamente, podríamos lamentarlo al ocurrir siniestros que afectarían lo que más nos duele: la pérdida de vidas humanas. 


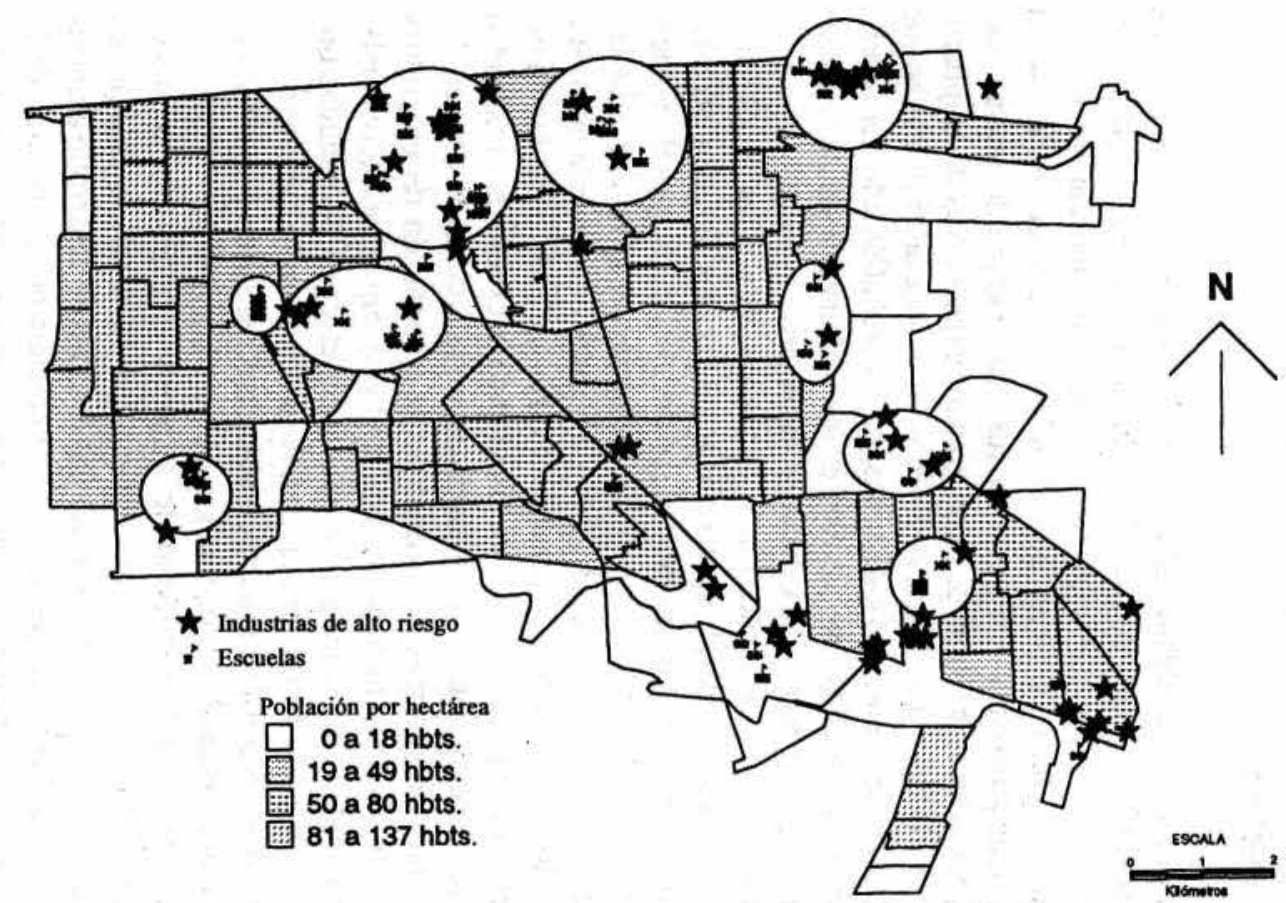

Figura 5. Escuelas cercanas a industrias de alto riesgo en Mexicali, B.C.

FUENTE: Instituto de investigaciones Sociales, UABC. 


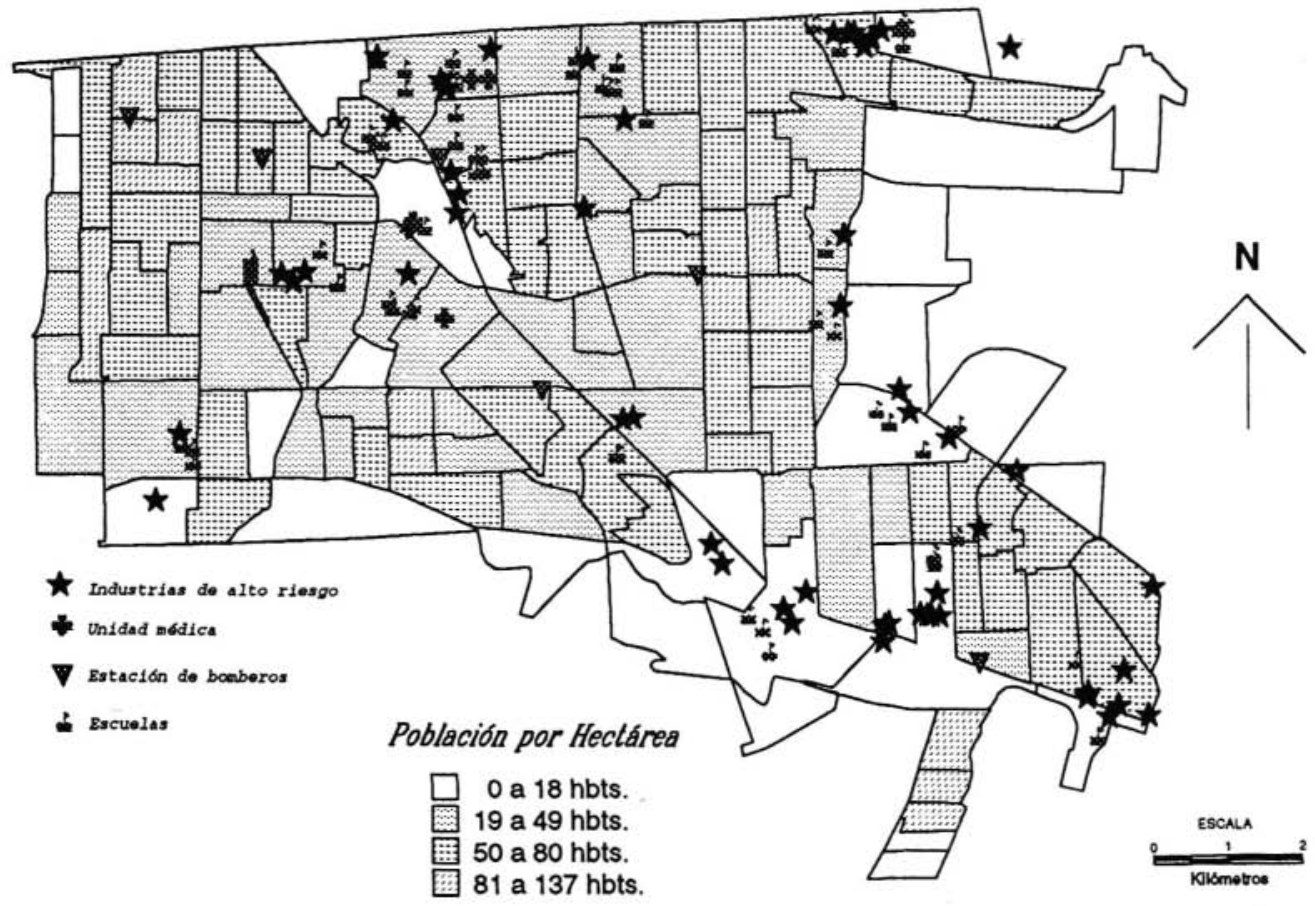

Figura 6. Industrias de alto riesgo y servicios en Mexicali, B.C. FUENTE: Instituto de investigaciones Sociales, UABC. 


\section{BIBLIOGRAFÍA}

ÁLVAREZ, L. Juan y Alejandro Mungaray. 1986. "Maquiladoras, Política Económica y Medio Ambiente en la Frontera Norte de México". En: Álvarez, L. Juan y Víctor M. Castillo (coords.). Ecología y frontera. Universidad Autónoma de Baja California.

CANACINTRA. 1985. Directorio industrial fronterizo. Cámara Nacional de la Industria de Transformación. Mexicali, B. Cfa.

Dirección General de Estadística. 1940. VI Censo general de población. México.

Dirección General de Estadística. 1950. VII Censo general de población $y$ vivienda. México.

Dirección General de Estadística. 1960. VIII Censo general de población y vivienda. México.

ELLIOT, Bart E. 1995. "Cities Turn to GIS to Enhance Efficiency and Responsiveness". Earth Observation Magazine, agosto.

ESTES, John, et al. 1987. "Coordinating Hazardous Waste Management. Activities Using Geographic Information Systems". International Journal of Geographic Information Systems, vol. 1, núm. 4.

FEDRA, Kurt. 1989. "Computer-Based Information and Decision Support Systems for Management of Hazardous Substances and Industrial Risk". En: Maltezou, Sonia P.; Asit K. Biswas and Hans Sutter (eds.). Hazardous Waste Management. Tycooly. Londres, 1989.

HUXHOLD, William E. 1991. An Introduction to Urban Geographic Information Systems. Oxford University Press. Nueva York.

INEGI. 1990. XI Censo general de población y vivienda. México.

INEGI. Censos económicos 1994. Versión disco compacto.

McMaster, Robert B. 1990. "Modeling Community Vulnerability to Hazardous Materials Using Geographic Information Systems". En: Peuquet, Donna J. y Duane F. Marble. Introductory Readings in Geographic Information Systems. Taylor and Francis. Londres.

SÁNCHEZ, Roberto A. 1989. "Contaminación de la Industria Fronteriza: Riesgos para la Salud y el Medio Ambiente". En: GonzálezAréchiga, Bernardo y Rocío Barajas Escamilla (coords.). Las maquiladoras: Ajuste estructural y desarrollo regional. El Colegio de la Frontera, México.

- 1991, "Otra Manera de Ver a la Maquiladora: Riesgos en el Medio Ambiente y en la Salud". En: González-Aréchiga, Bernardo y José Carlos Ramírez (coords.). Subcontratación y empresas trasnacionales. Apertura y reestructuración de la maquiladora. COLEF-Fundación Friedrich Ebert. México. 
SÁNDEZ Pérez, Agustín. 1988. "El Proceso de Industrialización en Baja California”. En: Estudios Fronterizos, núm. 15-16, IIS-UABC, enero-abril y mayo-agosto, Mexicali, B.C.

SÁNDEZ Pérez, Agustín, Ana María Avilés Muñoz y Guillermo Álvarez de la Torre. 1993. "Industrial Energy Load Study in Mexicali, Baja Califomia". Instituto de Investigaciones Sociales. UABC, (mimeo). STAR, Jeffrey y John Estes. 1990. Geographic Information Systems. Prentice Hall. New Jersey,

TAMAYO, Jesús. 1992. "Breve Balance y Perspectiva de la Industria Maquiladora de Exportación". En: Estudios Fronterizos, núm. 27-28, enero-abril y mayo-agosto, IIS-UABC.

WRIGHT, Richard, George Hepner y Clifton G. Metzner Jr. 1993. A United States-Mexico Border Environmental Geographic Information System. Institute for Regional Studies of the Californias. San Diego State University. San Diego, Ca.

\section{Diarios consultados}

El Economista, 14 de diciembre de 1995.

La Crónica de Baja California, 10 de enero de 1996.

La Crónica de Baja California, 21 de enero 21 de 1996.

La Crónica de Baja California, 18 de febrero de 1996.

La Voz de la Frontera, 6 de junio de 1990.

La Voz de la Frontera, 10 de junio de 1990.

La Voz de la Frontera, 21 de junio de 1992.

La Voz de la Frontera, 28 de mayo de 1996

Periódico Oficial del Estado de Baja California, 24 de junio de 1994. 